\title{
Prevention of Anti-BRAF-Induced Phototoxicity: not Just a Question of Photoprotector
}

\author{
M Cormerais $^{1}$, A Boisrobert ${ }^{2}$, H Dutartre ${ }^{2}$, A Khammari $^{2}$ and B Dreno ${ }^{2^{*}}$ \\ ${ }^{1}$ Service de Dermatologie, CHU Pontchaillou, France \\ ${ }^{2}$ Service de Dermatologie, $\mathrm{CHU}$ de Nantes, France
}

*Corresponding author: B Dreno, Service de Dermatologie, CHU de Nantes, Place Alexis Ricordeau 44093 Nantes, France

\section{Letter to Editor}

Targeted therapies changed the prognosis of metastatic melanoma. Flaherty \& al. and the BRIM-II study found a response rate between $53-81 \%$ and a median progression-free survival of about seven months $[1,2]$. The BRIM-III study reported a median survival of 13.6 months and a 6-month survival rate of $84 \%$ with Vemurafenib versus 9.7 months and 64\% with dacarbazine [3]. The UVA-phototoxicity induced by Vemurafenib through a decrease in minimal erythema dose [4,5], was found respectively in 16\% (1), $52 \%$ (2) and $40 \%$ (3) of patients in these 3 studies. Although the benefit of sunscreen has been mentioned (4), to our knowledge, no efficacy study with an anti- UVA sunscreen has been conducted. This study assesses the benefit of therapeutic education associated with a photoprotector in a real-life setting. Patients with unresectable stage IIIC or IV BRAFV600E-mutated melanoma, treated with Vemurafenib 960mg twice daily, were included in the Dermatology department of Nantes University Hospital. Patients received 30mn training session performed by a nurse on the sunscreen during which they received free SPF50+ anti UVA $(\leqslant 3)$ photoprotector.

Regular follow-up was as well organized. After 12 weeks, patients were asked to self-asses: average daily application, application areas, tolerance to the treatment, by looking at redness level, burning sensation and/or pruritus sensation (classified in absent, mild, moderate or severe). The nurse assessed compliance at each follow-up. Patients were classified in group 1 with no or mild phototoxicity and in group 2 with moderate or severe phototoxicity. Only patients who applied photoprotector at least twice daily were considered compliant. Twenty-seven patients with a mean age of 56.2 years (32-89 years) were included. Fifteen patients were classified in group 1 and 12 in group 2 based on their self-reporting, including 6 patients with moderate phototoxicity and 6 patients with severe phototoxicity. Fourteen patients were considered compliant. Nurse questioning revealed that patients did not evaluate their observance correctly, and that only 2 patients of the group 2 were actually observant. The 10 other patients confessed regular omissions. Therefore in 25 out of the 27 patients, no significant phototoxicity on sun protected areas was observed.

A non-significant increase in phototoxicity (group 2) was observed in non-compliant patients (OR=2.04, 95\%CI $(0.35 ; 12.89)$, $\mathrm{p}=0.45$ ). There was a non-significant difference in phototoxicity according to the age $(\mathrm{OR}=0.63,95 \% \mathrm{CI}(0.10 ; 3.7), \mathrm{p}=0.70)$ or to the gender $(\mathrm{OR}=0.80,95 \% \mathrm{CI}(0.130 ; 5.23), \mathrm{p}=1)$. Anti-BRAF, photosensitivity and sunscreen There was a non-significant trend toward a decreased phototoxicity in patients with skin phototype $3(\mathrm{OR}=0.2621,95 \% \mathrm{CI}(0.10 ; 3.7), \mathrm{p}=0.70)$ and in women $(\mathrm{OR}=0.24$, $95 \%$ CI $(0.02 ; 1.76), p=0.22)$. Despite a therapeutic education and a free photoprotector, only 55\% of patients reported no or mild phototoxicity and 52\% were observant Various factors could explain these results, including a possible over-reporting of phototoxicity; Indeed, the phototoxicity was self-assessed by patients themselves since the treatment was taken at home and in addition, a strict definition of phototoxicity was used. Sunscreen has to be applied several times a day and in a context of anti-cancer treatment, it could appear of secondary importance compared to anti-cancer treatment explaining the low observance. In addition, UVAs pass through clouds and windows [6].

Patients should understand that photoprotection is needed daily in any weather and in any place. The absence of refund could be an additional barrier. A sun protection kit with Vemurafenib could be interesting. Phototoxicity appeared only on under-treated areas, except in 2 patients of group 2. This emphasizes the link between efficacy and compliance with treatment and the importance of therapeutic education. Klaeger et al. [7] reported that Vemurafenib could inhibit ferro chelatase responsible for an accumulation of Protoporphyrin. In analogy to Erythropoietic Protoporphyrin, light absorption induces a production of Reactive oxygen species and inflammatory process with complement activation and mast cell degranulation explaining vasodilation and edema described in phototoxic reactions [8]. Alfamelanotide, an analog of alpha- 
melanocyte-stimulating hormone approved in Erythropoietic Protoporphyrin, could theoretically be discussed in combination with Vemurafenib [9]. However, carcinogenic potential is unknown [10]. In conclusion, the systematic prescription of an anti-UVA/ UVB photoprotector is insufficient to protect against Vemurafenibinduced phototoxicity. It should be accompanied by therapeutic education repeated during treatment. Cutaneous prevention is not a central concern for patients with metastatic melanoma. In this context, protective clothing remains essential.

\section{Acknowledgment}

We thank La Roche Posay International laboratories for providing the photoprotector. There is no other conflict of interest to report for this study.

\section{References}

1. Flaherty, Keith T, Igor Puzanov, Kevin B Kim, Antoni Ribas, et al. (2010) 'Inhibition of Mutated, Activated BRAF in Metastatic Melanoma'. New England Journal of Medicine 363 (9): 809-819.

2. Sosman, Jeffrey A, Kevin B Kim, Lynn Schuchter, Rene Gonzalez, et al. (2012) 'Survival in BRAF V600-Mutant Advanced Melanoma Treated with Vemurafenib'. New England Journal of Medicine 366 (8): 707-714.

3. McArthur, Grant A, Paul B Chapman, Caroline Robert, James Larkin, et al. (2014). 'Safety and Efficacy of Vemurafenib in BRAFV600E and BRAFV600K Mutation-Positive Melanoma (BRIM-3): Extended Followup of a Phase 3, Randomised, Open-Label Study'. The Lancet Oncology 15 (3): 323-332.
4. Dummer, Reinhard, Jeannine Rinderknecht, Simone M Goldinger (2012) 'Ultraviolet A and Photosensitivity during Vemurafenib Therapy'. New England Journal of Medicine 366 (5): 480-481.

5. Brugière C, A Stefan, C Morice, E Cornet, A Moreau, et al. (2014) 'Vemurafenib Skin Phototoxicity Is Indirectly Linked to Ultraviolet A Minimal Erythema Dose Decrease'. British Journal of Dermatology 171(6): 1529-1532.

6. Sinha R, K Edmonds, JA Newton Bishop, ME Gore, J Larkin, et al. (2012) 'Cutaneous Adverse Events Associated with Vemurafenib in Patients with Metastatic Melanoma: Practical Advice on Diagnosis, Prevention and Management of the Main Treatment-Related Skin Toxicities Cutaneous Adverse Events with Vemurafenib: Practical Advice'. British Journal of Dermatology 167(5): 987-994.

7. Klaeger, Susan, Bjoern Gohlke, Jessica Perrin, Vipul Gupta, et al. (2016) 'Chemical Proteomics Reveals Ferrochelatase as a Common Off-Target of Kinase Inhibitors'. ACS Chemical Biology 11(5): 1245-54.

8. Lecha, Mario, Hervé Puy, Jean Charles Deybach (2009) 'Erythropoietic Protoporphyria'. Orphanet Journal of Rare Diseases 4: pp19.

9. Biolcati G, E Marchesini, F Sorge, L Barbieri, X Schneider Yin, et al. (2015) 'LongTermObservational Study of Afamelanotide in 115 Patients with Erythropoietic Protoporphyria'. British Journal of Dermatology 172 (6): 1601-1612.

10. Kim, Esther S, Karly P Garnock Jones (2016) 'Afamelanotide: A Review in Erythropoietic Protoporphyria'. American Journal of Clinical Dermatology 17(2): 179-85.

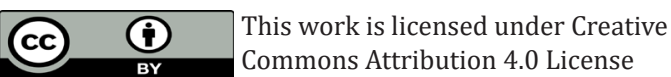

To Submit Your Article Click Here:

Submit Article

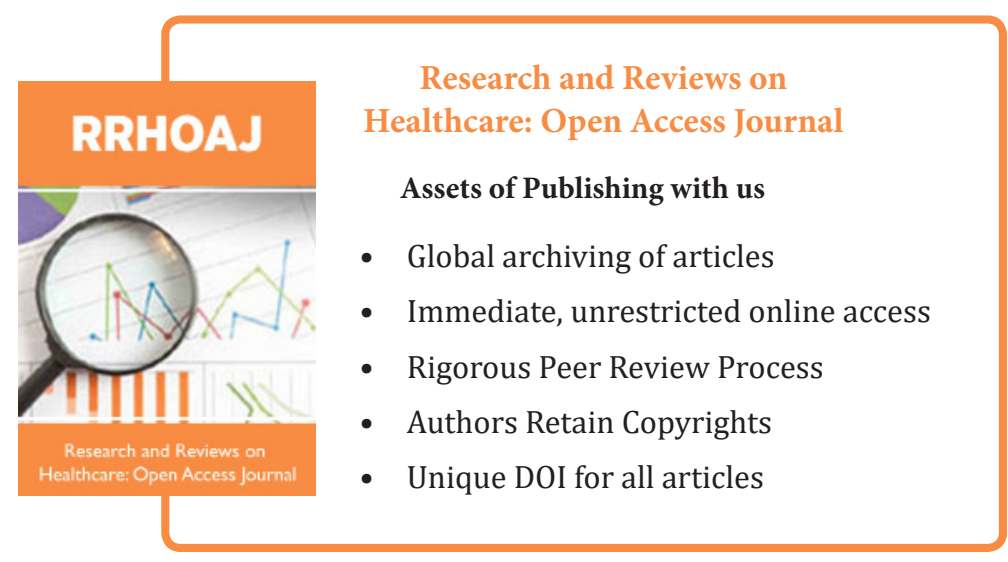

